\title{
SEMI-FIELD-LIKE AFFINE PLANES
}

\author{
by MICHAEL J. KALLAHER $\dagger$
}

(Received 27 October, 1975)

1. Introduction. Walker [11] describes a new class of translation planes $W(q)$ of order $q^{2}, q \equiv 5(\bmod 6)$, with kernel $G F(q)$. A plane in this class has several interesting properties, but we shall be only interested in the following ones possessed by its collineation group $G$ : (i) $G$ is transitive on the affine points of $W(q)$, and (ii) $G$ fixes a point $\mathscr{V}_{\infty} \in l_{\infty}$, the line at infinity of $W(q)$, and is transitive on the other points of $\ell_{\infty}$. The smallest member of this class, $W(5)$, also satisfies: (iii) for an affine point $\mathcal{O}$, the subgroup $G_{\mathscr{O}}$ is transitive on the affine points $\neq \mathcal{O}$ of the line $\mathcal{O} \mathscr{V}_{\infty}$. Note also that since $W(5)$ is a translation plane, replacing $G$ with $G_{\mathfrak{O}}$ in (ii) we get a fourth property, call it (iv), satisfied by $G$. (See Lemma 2.)

All semi-field planes satisfy properties (i) and (ii), but the planes $W(q)$ are the only known translation planes of odd order satisfying (i) and (ii) which are not semi-field planes. For characteristic 2, the Lüneburg planes have a collineation group $G$ satisfying (i) and (ii). The plane $W(5)$ is the only known non-semi-field translation plane that satisfies (i), (ii), and (iii). This article is the report of an attempt to characterize the plane $W(5)$ using properties (i), (ii), (iii).

MAIN THEOREM. If $\mathfrak{A}$ is a translation plane of finite order $p^{r}$ satisfying (i), (ii), (iii), and $\mathfrak{A}$ is not a semi-field plane, then $\mathfrak{A}$ must also satisfy the following:

(1) 2 divides $r$;

(2) the collineation group $G$ of $\mathfrak{A}$ is non-solvable if $p^{r} \neq 2^{6}$;

(3) $\mathfrak{Y}$ has even dimension $d \geqq 4$ over its kernel if $p^{r} \neq 5^{2}, 11^{2}, 2^{6}, 3^{4}$.

This follows from Lemma 2, Definitions 2 and 3, Theorem 8, Corollary 8.1, and Theorem 9.

Before proving these results, we consider in section 2 affine planes satisfying properties (i), (ii), (iii), (iv). Such planes are called $W A$-planes. It follows from Theorem (3.7) in [6] that a $W A$-plane of odd order is either a translation plane or a dual translation plane. Thus, in considering $W A$-planes of odd order we may restrict ourselves to those which are translation planes; naturally, we call such planes $T W A$-planes. The plane $W(5)$ is a $T W A$-plane. We then, in sections 4 and 5, prove the conclusions (1), (2), (3) given above for $T W A$-planes in general. They then also hold for $W A$-planes of odd order (Corollary 8.2 and Corollary 9.1).

A second reason for this investigation lies in the following problem proposed by Ostrom [10]: Does there exist a translation plane of non-square order that is not a semi-field plane but whose collineation group $G$ satisfies (ii)? In a private communication Ostrom has suggested that such examples may be constructible by the techniques Walker used in constructing the planes $W(q)$. The present investigation indicates that this attack on his problem may not work.

† The author was supported in part by NSF Grant No. MPS75-05260 and in part by a Fulbright fellowship, which the author held at the Fachbereich Mathematik der Universität, Kaiserslautern, during the academic year 1975-76.

Glasgow Math. J. 18 (1977) 113-123.

$\mathbf{H}$ 
Finally, as indicated above, there is a connection between this article and the article [6]. In the terminology of [6], a $W A$-plane is an affine plane of rank $(r, s, 2)$ admitting a block orbit of degree 1.

The reader is expected to be familiar with the terminology of projective and affine planes as exhibited in [3] and also with the theory of permutation groups as given in [12].

\section{Definitions and preliminary results.}

DefinITION 1. A $W$-plane is an affine plane $\mathfrak{A}$ of finite order $n$ possessing a collineation group $G$ having the following properties:

(i) $G$ is transitive on the affine points of $\mathfrak{A}$,

(ii) $G$ fixes a point $\mathscr{V}_{\infty} \in l_{\infty}$, the line at infinity of $\mathfrak{A}$, and is transitive on the remaining points of $l_{\infty}$.

The collineation group $G$ is called a $W$-group of $\mathfrak{A}$ and $\mathscr{V}_{\infty}$ is called the special point of $\mathfrak{A}$ with respect to $G$.

Examples. (1). Every semi-field plane $\mathfrak{A}$ satisfies (i) and (ii) where $G$ is the collineation group generated by the translations and the affine elations fixing the point $(\infty)$. If $\mathfrak{A}$ is not Desarguesian, $G$ can be taken as the full collineation group.

(2) Walker [11] discovered a class of translation planes $W(q), q \equiv 5(\bmod 6)$, satisfying conditions (i) and (ii). These planes and their duals are the only known planes of odd order satisfying (i) and (ii) which are not semi-field planes.

(3) The Lüneburg planes of order $2^{2 r}, r$ odd and at least 3 , and their duals possess a collineation group satisfying (i) and (ii). It is also possible that the planes derived from the dual Lüneburg planes have a collineation group satisfying (i) and (ii). (See Dembowski [3, p. 251].)

Before moving on to the principal object of study in this article, we make two observations about $W$-planes.

LEMMA 1 . The dual of $a$ W-plane is a W-plane.

Proof. In dualizing $\mathfrak{A}$ to get the dual plane $\mathfrak{Q}^{\prime}$ we take $\mathscr{V}_{\infty}^{\prime}$, the dual of the point given in (ii), to be the line at infinity of $\mathfrak{U}^{\prime}$ and $\mathfrak{l}_{\infty}^{\prime}$ is the special point of $\mathfrak{I}^{\prime}$. Then the affine points of $\mathfrak{H}^{\prime}$ correspond to the affine lines of $\mathfrak{U}$ not through $\mathscr{V}_{\infty}$. The lemma then follows from a result of A. Wagner. (See $[3,4.4 .24]$.)

The smallest Walker plane, $W(5)$, satisfies in addition to properties (i) and (ii) the following condition:

(iii) for an affine point $\mathcal{O}$ of $\mathfrak{A}, G_{\mathscr{O}}$ is transitive on the affine points $\neq \mathcal{O}$ of the line $\mathscr{O} \mathscr{V}_{\infty}$. Also, in the Walker planes the $W$-group contains the group of translations. With regard to these two facts we make the following observation.

LEMMA 2. Let $\mathfrak{A}$ be a W-plane with a $W$-group $G$ and special point $\mathscr{V}_{\infty}$. If $\mathfrak{A}$ is a translation plane and $G$ contains the group of translations, or if $\mathfrak{\mathfrak { T }}$ is a dual translation plane and $G$ contains the group of dual translations, then $\mathfrak{A}$ and $G$ satisfy: 
(iv) for an affine point $\mathcal{O}$ of $\mathfrak{U}$, the group $G_{\mathfrak{O}}$ is transitive on the points of $l_{\infty}-\left\{\mathscr{V}_{\infty}\right\}$.

Proof. Assume $\mathfrak{U}$ is a translation plane and $G$ contains the group $T$ of translations; then $G=T G_{\mathscr{Q}}$ and, because $T$ fixes every point of $\ell_{\infty}, G$ and $G_{\mathscr{\vartheta}}$ induce the same permutation group on $l_{\infty}$. If $\mathfrak{U}$ is a dual translation plane and $G$ contains the group of dual translations, then $G$ contains the group $E$ of dual translations with centre $\mathscr{V}_{\infty}$ and axis $\mathcal{O} \mathscr{V}_{\infty}$ which is transitive on $l_{\infty}-\left\{\mathscr{V}_{\infty}\right\}$

REMARK. The planes coordinatized by the twisted fields of Albert [1] satisfy (iii); however, the Lüneburg planes and their duals do not.

Because of Lemma 2 and the plane $W(5)$, we will restrict ourselves to the study of the following subclass of $W$-planes.

DEFINITION 2. A $W A$-plane is an affine plane $\mathfrak{U}$ of finite order $n$ possessing a collineation group $G$ satisfying properties (i), (ii), (iii), and (iv). The group $G$ is called a WA-group.

Our first justification for studying this class is our first theorem.

THEOREM 1. If $\mathfrak{H}$ is a WA-plane of odd order $n$ with WA-group $G$, then either $\mathfrak{A}$ is a translation plane and $G$ contains the translations or $\mathfrak{A}$ is a dual translation plane and $G$ contains the dual translations.

Proof. This is just a restatement of Theorem (3.7) in [3] since a $W A$-plane is an affine plane of rank $(r, s, 2)$ admitting a block orbit of degree 1 .

As a corollary we can prove that for odd order the class of $W A$-planes is closed under duality.

THEOREM 2. The dual of a WA-plane of odd order is a WA-plane.

Proof. Let $\mathfrak{U}$ be a $W A$-plane of odd order $p^{r}$ with $W A$-group $G$ and special point $\mathscr{V}_{\infty}$. To show that the dual $\mathfrak{U}^{\prime}$ is a $W A$-plane, it is sufficient to prove the following:

(i') $G$ is transitive on the affine lines of $\mathfrak{A}$ not through $\mathscr{V}_{\infty}$;

(ii') $G$ fixes $l_{\infty}$;

(iii') for every affine line $\ell$ of $\mathfrak{U}$ with $\mathscr{V}_{\infty} \notin \ell$, the group $G_{\ell}$ is transitive on the affine lines through $\mathscr{V}_{\infty}$

$\left(i v^{\prime}\right)$ for every affine line $\ell$ of $\mathfrak{A}$ with $\mathscr{V}_{\infty} \notin l$, the group $G_{\ell}$ is transitive on the affine lines $\neq \ell$ through $\ell \cap l_{\infty}$.

(ii') is obvious and (i') follows from 4.4 .24 of [3] as in the proof of Lemma 1.

Assume first that $\mathfrak{U}$ is a translation plane. Let $\mathfrak{l}$ be an affine line of $\mathfrak{U}$ not through $\mathscr{V}_{\infty} ; G_{l}$ contains the group $T\left(\mathscr{U}_{\infty}\right)$ of translations with centre $\mathscr{U}_{\infty}=\ln \ell_{\infty}$. Now, $T\left(\mathscr{U}_{\infty}\right)$ fixes $\ell$ and is transitive on the affine points of $\ell$; since $T\left(\mathscr{U}_{\infty}\right)$ also fixes $\mathscr{V}_{\infty}$, (iii') holds. To prove (iv'), let $\mathcal{O}$ be an affine point on $\ell$ and consider $G_{\ell, O}=G_{\iota_{,} \mathscr{U}_{\infty}}$, where $\mathscr{U}_{\infty}=\ell_{\cap} \ell_{\infty}$. We have the following equations by (iii) and (iv):

$$
\left|G_{\mathscr{O}}\right|=p^{r}\left|G_{\mathcal{O}, \mathscr{U}_{\infty}}\right|, \quad\left|G_{\mathscr{O}}\right|=\left(p^{r}-1\right)\left|G_{\mathscr{O}, \mathscr{P}}\right|,
$$


where $\mathscr{P}$ is an affine point of $\mathscr{O} \mathscr{V}_{\infty}$, with $\mathscr{P} \neq \mathcal{O}$. Thus

$$
\left|G_{\mathscr{O}, \mathscr{F}}\right|=p^{r} k, \quad\left|G_{\mathscr{O}, \mathscr{U}_{\infty}}\right|=\left(p^{r}-1\right) k,
$$

for some integer $k \geqq 1$.

Let $H=G_{\mathscr{O}, \mathscr{q}_{\infty}}$. Then $H_{\mathscr{P}} \leqq G_{\mathscr{O}, \mathscr{P}}$ and thus $\left|H_{\mathscr{P}}\right| \mid p^{r} k$. On the other hand, $\left|H_{\mathscr{F}}\right| \mid\left(p^{r}-1\right) k$. Thus $\left|H_{\mathscr{F}}\right| \mid k$ and hence for the orbit $\Phi$ of $\mathscr{P}$ under $H$, we have $\left(p^{r}-1\right)|| \Phi \mid$ since $|\Phi|=$ $|H|\left|H_{\mathscr{g}}\right|^{-1}$. It follows that $\Phi$ consists of the affine points of $\mathcal{O} \mathscr{V}_{\infty}$ other than $\mathcal{O}$. Thus $H=G_{\mathcal{O}, \mathscr{\Psi}_{\infty}}=G_{\mathcal{O}, l}$ is transitive on the affine points of $\mathcal{O} \mathscr{V}_{\infty}$ other than $\mathcal{O}$ and hence (iv') is satisfied.

Assume now that $\mathfrak{A}$ is a dual translation plane. Let $l$ be an affine line of $\mathfrak{A}$ not through $\mathscr{V}_{\infty}$. By (i'), (i), and (iii) we have

$$
|G|=p^{2 r}\left|G_{l}\right|, \quad|G|=p^{2 r}\left|G_{\mathscr{O}}\right|=p^{3 r}\left|G_{\mathscr{O}, q_{l \infty}}\right|, \quad|G|=p^{r}\left|G_{\mathscr{q}_{\infty}}\right|,
$$

where $\mathcal{O}$ is an affine point and $\mathscr{U}_{\infty}$ a point on $\ell_{\infty}$ with $\mathscr{U}_{\infty} \neq \mathscr{V}_{\infty}$. Thus

$$
\left|G_{l}\right|=p^{r}\left|G_{\theta, \mathscr{U}_{\infty}}\right|, \quad\left|G_{\mathscr{U}_{\infty} \mid}\right|=p^{2 r}\left|G_{\theta_{0, \varkappa_{\infty}}}\right|, \quad\left|G_{\mathscr{U}_{\infty}}\right|=p^{r}\left|G_{l}\right| .
$$

Now, let $\mathscr{O} \in l$ and $\mathscr{U}_{\infty}=\ln l_{\infty}$; then $G_{\mathscr{O}, \mathcal{U}_{\infty}} \leqq G_{\ell} \leqq G_{\mathscr{U}_{\infty}}$ and $G_{\mathcal{O}, \mathcal{U}_{\infty}}=G_{\mathscr{O}, l}$. If $\Phi$ is the orbit of $\mathcal{O}$ under $G_{l}$, then

$$
\left|G_{\ell}\right|=|\Phi|\left|G_{0, \ell}\right|=|\Phi|\left|G_{0, w_{\infty}}\right| \text {. }
$$

Hence from the first equality of (2.3), $|\Phi|=p^{r}$; therefore, $G_{\ell}$ maps $\mathcal{O}$ into $p^{r}$ distinct affine points. Since $G_{\ell}$ fixes $\ell$ and $\mathcal{O} \in l, G_{\ell}$ must be transitive on $\ell$. It follows that $G_{\ell}$ is transitive on the lines through $\mathscr{V}_{\infty}$. This proves (iii').

For (iv'), let $\mathscr{P}$ be an affine point on $\mathscr{O} \mathscr{V}_{\infty}, \mathscr{P} \neq \mathscr{O}$. We have the equalities

$$
\left|G_{0}\right|=\left(p^{r}-1\right)\left|G_{\mathcal{O}, \mathscr{g}}\right|, \quad\left|G_{\mathscr{U}_{\infty}}\right|=p^{r}\left|G_{\mathcal{U}_{\infty}, 0 V_{\infty}}\right|,
$$

since $G_{\mathcal{O}}$ is transitive on $\mathcal{O} \mathscr{V}_{\infty}-\{\mathcal{O}\}$, and $G_{l} \leqq G_{\mathscr{q}_{\infty}}$ implies $G_{\mathscr{U}_{\infty}}$ is also transitive on the affine lines through $\mathscr{V}_{\infty}$. By (2.2) we must have

$$
\left|G_{\mathfrak{q}_{\infty}, \mathrm{V}_{\infty}}\right|=\left(p^{r}-1\right)\left|G_{0, g}\right| \text {. }
$$

Since $\mathfrak{A}$ is a dual translation plane, $G_{\mathscr{O},}$ contains the group $E$ of elations with axis $O \mathscr{V}_{\infty}$; hence

$$
\left|G_{\mathscr{O}, \mathscr{P}}\right|=p^{r}\left|G_{0, \mathscr{P}, \mathscr{q}_{\infty}}\right|
$$

and

$$
\left|G_{\mathscr{H}_{\infty}, \text { Or }}\right|=p^{r}\left(p^{r}-1\right)\left|G_{\mathscr{O}, \mathscr{P}, \mathbb{U}_{\infty}}\right| \text {. }
$$

Furthermore,

$$
\left|G_{\mathscr{u}_{\infty}, O V_{\infty}}\right|=p^{r}\left|G_{\mathscr{q}_{\infty}, \mathcal{O}}\right|
$$

since $G_{\mathscr{U}_{\infty}, \mathscr{V}_{\infty}}$ contains the group $T\left(\mathscr{V}_{\infty}\right)$ of translations with centre $\mathscr{V}_{\infty}$ and $G_{\mathscr{U}_{\infty}, \mathcal{O}}=$ $T\left(\mathscr{V}_{\infty}\right) G_{\mathscr{q}_{\infty}, 0 V_{\infty}, 0}$. Equations (2.5) and (2.6) give

$$
\left|G_{\mathscr{U}_{\infty}, \mathcal{O}}\right|=\left(p^{r}-1\right)\left|G_{\mathcal{O}, \mathscr{Q}, \mathscr{U}_{\infty}}\right| \text {. }
$$


If $\Phi$ is the orbit of $\mathscr{P}$ under $G_{\mathscr{q}_{\infty}, \mathcal{O}}$, then

$$
|\Phi|=\left|G_{\mathscr{U}_{\infty}, \mathcal{O}}\right|\left|G_{\mathscr{Q}_{\infty}, \mathcal{O}, g}\right|^{-1}=p^{r-1}
$$

by equation (2.7). Therefore $G_{\mathscr{Q}_{\infty}, \mathcal{O}}$ is transitive on the affine points $\neq \mathcal{O}$ of $\mathcal{O} \mathscr{F}_{\infty}$. It follows that $G_{\mathfrak{u}_{\infty}, \mathcal{O}}$ is transitive on the affine lines $\neq \ell=0 \mathscr{U}_{\infty}$ through $\mathscr{U}_{\infty}$. Since $G_{\mathfrak{U}_{\infty}, \mathcal{O}} \leqq G_{\ell}$, (iv') is satisfied.

We close this section with a theorem that will be used frequently in the succeeding sections. This theorem is a slight modification of Theorem 1 in [7]. By the translation complement of a translation plane we mean the group of collineations fixing an affine point $\mathcal{O}$. (See Ostrom [10].) Considering Lemma 2 and Theorem 1 in [7], it is immediately clear that they hold for any subgroup of the translation complement, not just the translation complement. Secondly, in part (b) of the conclusion of Lemma 2, the values $q=4,5$ do not really occur since part (b) reduces to part (a) for these two values. This is obvious for $q=5$ and it holds for $q=4$ since $P S L(2,4)=P S L(2,5)$. Thus, in part (b) of Theorem 1 the values $q=4,5$ do not appear. Hence we have the following theorem.

THEOREM 3. Let $\mathfrak{U}$ be a finite translation plane of order $q^{2}, q=p^{r}$ with $p$ a prime, and having kernel $\bar{K}=G F(q)$. Let $G$ be a non-solvable collineation group of $\mathcal{U}$ fixing an affine point $\mathcal{O}$ and an affine line $l$ with $\mathcal{O} \in l$ such that $G$ is transitive on the affine points of $\ell-\{\mathcal{O}\}$. Assume also that $G$ contains the group $K$ of $\left(\mathcal{O}, \ell_{\infty}\right)$-homologies. If $H$ is the group of collineations in $G$ fixing $\ell$ pointwise, then $G / H$ is a subgroup of $\Gamma L(2, q)$ and either

(a) $G / H$ contains $S L(2, q),|G / H|=\frac{1}{2} k q\left(q^{2}-1\right)(q-1)$ with $k \mid 2$ or

(b) $G / H$ contains $S L(2,5),|G / H|=60 k(q-1)$ with $k \mid 2$ and $q=9,11,19,29$ or 59.

3. $W A$-planes of order $p^{2}, p$ a prime. We turn to the question of which translation planes are $W A$-planes. In this section we give some preliminary facts about such planes and consider the case when the order is $p^{2}, p$ a prime; in the next section we consider the case when the $W A$-group is solvable, and in the last section we look at $W A$-planes having dimension 2 over their kernel. Because of Theorems 1 and 2 we need only concern ourselves, in the case of odd order, with $W A$-planes which are translation planes. However, most of the remaining results hold for translation planes of even order, and we include them in the discussion. We thus make the following definition.

Definition 3. A TWA-plane is a $W A$-plane that is a translation plane.

Lemma 3. Let $\mathfrak{U}$ be a TWA-plane of order $p^{r}$, $p$ a prime, $G$ a WA-group of $\mathfrak{A}$, and $\mathscr{V}_{\infty}$ the special point of $\mathfrak{U}$ with respect to $G$. If $\mathcal{O}$ is an affine point of $\mathfrak{U}, \mathscr{U}_{\infty}$ a point in $\ell_{\infty}-\left\{\mathscr{V}_{\infty}\right\}$, and $\mathscr{P}$ an affine point on $\mathcal{O} \mathscr{V}_{\infty}-\{\mathcal{O}\}$, then the following hold:

$$
\begin{aligned}
|G| & =p^{2 r}\left|G_{\mathcal{O}}\right|, \\
\left|G_{\mathcal{O}}\right| & =p^{r}\left|G_{\mathcal{O}, \mathscr{U}_{\infty}}\right|=\left(p^{r}-1\right)\left|G_{\mathcal{O}, \mathscr{g}}\right|, \\
\left|G_{\mathcal{O}, \mathscr{U}_{\infty}}\right| & =\left(p^{r}-1\right)\left|G_{\mathcal{O}, \mathscr{T}, U_{\infty}}\right| .
\end{aligned}
$$


Proof. (3.1) and (3.2) follow from the definition of a $W A$-plane and (3.3) follows from the the next lemma.

LemMa 4. Let $\mathfrak{U}$ be a TWA-plane of order $p^{r}, G$ a WA-group of $\mathfrak{U}$, and $\mathscr{V}_{\infty}$ the special point of $\mathfrak{U}$ with respect to $G$. If $\mathcal{O}$ is an affine point of $\mathcal{U}$ and $\mathscr{U}_{\infty}$ a point of $\ell_{\infty}$ with $\mathscr{U}_{\infty} \neq \mathscr{V}_{\infty}$, then $G_{\mathcal{O}, \mathscr{U}_{\infty}}$ is transitive on the affine points of $\mathcal{O} \mathscr{V}_{\infty}-\{\mathcal{O}\}$.

Proof. A proof of this lemma was given during the proof of Theorem 2; see the third paragraph of that proof.

Let $(Q,+, \cdot)$ be a quasi-field coordinatizing the $T W A$-plane $\mathfrak{A}$ with $\mathscr{V}_{\infty}=(\infty), \mathcal{O}=(0,0)$, where $\mathscr{V}_{\infty}$ is the special point with respect to a $W A$-group $G$ of $\mathfrak{A}$. Since $G_{0}$ fixes the line $\mathcal{O} \mathscr{V}_{\infty}, G_{\mathcal{O}}$ induces a group $\bar{G}$ of linear transformations on $(Q,+)$ as a vector space over the prime field $G F(p)$. In fact, if $\sigma \in G_{\mathscr{o}}$ then $\sigma$ induces the linear transformation $\bar{\sigma}$ given by $y \bar{\sigma}=\bar{y}$ if and only if $(0, y) \sigma=(0, \bar{y})$. This gives the following theorem.

THEOREM 4. Let $\mathfrak{A}$ be a TWA-plane of order $p^{r}$ with WA-group $G$ and special point $\mathscr{V}_{\infty}$, and let $\mathcal{O}$ be an affine point of $\mathfrak{A}$. If $(Q,+, \cdot)$ is a quasi-field coordinatizing $\mathfrak{A}$ with $\mathscr{V}_{\infty}=(\infty)$ and $\mathcal{O}=(0,0)$, then $G_{\mathcal{O}}$ induces a group $\bar{G}$ of linear transformations lon $(Q,+)$ as a vector space over $G F(p)$. Furthermore, $\bar{G}$ is transitive on the non-zero vectors and $\bar{G}=G_{\mathcal{O}} / H$, where $H$ is the group consisting of all central collineations with axis $\mathcal{O} \mathscr{V}_{\infty}$ contained in $G$.

Remark. Note that by Lemma 4 , in Theorem 4 we could replace $G_{0}$ with the group $G_{\mathcal{O}, \mathscr{Q}_{\infty}}$ and the resulting statement is true. This fact will be used later.

THEOREM 5. Let $\mathfrak{U}$ be a TWA-plane of order $p^{r}$. If $\mathfrak{A}$ has a non-trivial homology whose axis is an affine line, then $\mathfrak{U}$ is a semi-field plane.

Proof. Let $G$ be a $W A$-group possessed by $\mathfrak{A}$ and $\mathscr{V}_{\infty}$ the special point. Assume $\sigma$ is a non-trivial homology of $\mathfrak{A}$ with axis $\ell, \ell$ an affine line. There are two possibilities: (1) $\mathscr{V}_{\infty} \in \mathfrak{l}$ and (2) $\mathscr{V}_{\infty} \notin l$. Assume first that $\mathscr{V}_{\infty} \in l$. We may assume the centre $\mathscr{C}$ of $\sigma$ lies on $l_{\infty}$; for if $\mathscr{C} \notin l_{\infty}$ then $\sigma$ moves $l_{\infty}$ and $\mathfrak{A}$ is Desarguesian [3, p. 130]. If $\mathcal{O}$ is an affine point on $l$, then by property (iv) for $G$ (see Lemma 2 ) for every point $\mathscr{D}_{\infty} \in l_{\infty}-\left\{\mathscr{V}_{\infty}\right\}, \mathfrak{U}$ has a non-trival homology with axis $\mathfrak{l}$ and centre $\mathscr{D}_{\infty}$. By Theorem 3 of Andre' [2], $\mathfrak{U}$ is $\left(\mathscr{V}_{\infty}, \mathfrak{l}\right.$-transitive and thus $\mathfrak{U}$ is a semi-field plane $[3$, p. 130].

Assume now that $\mathscr{V}_{\infty} \notin \ell$. If $\mathscr{V}_{\infty} \sigma=\mathscr{V}_{\infty}^{\prime} \neq \mathscr{V}_{\infty}$ then $\mathfrak{U}$ has a $W A$-group $G^{\prime}=\sigma^{-1} G \sigma$ with special point $\mathscr{V}_{\infty}^{\prime}$, and if $\tau \in G$ with $\mathscr{V}_{\infty}^{\prime} \tau=\ln \mathfrak{l}_{\infty}$ then $\tau^{-1} G^{\prime} \tau$ is a $W A$-group of $\mathfrak{A}$ with special point $\ln l_{\infty}$. By the argument in the first paragraph $\mathfrak{U}$ is a semi-field plane. (In fact, it is Desarguesian.)

Thus we may assume that $\mathscr{V}_{\infty}$ is the centre of $\sigma$. Let $\mathscr{O} \in l, \mathcal{O} \notin l_{\infty}$. By property (iv), for every line $\ell^{\prime}$ through $\mathcal{O}$ with $\ell^{\prime} \neq \mathcal{O} \mathscr{V}_{\infty}, \mathfrak{U}$ has a homology with axis $\ell^{\prime}$ and centre $\mathscr{V}_{\infty}$. By the dual of Theorem 3 in [2], $\mathfrak{H}$ is $\left(\mathscr{V}_{\infty}, \mathcal{O} \mathscr{V}_{\infty}\right)$-transitive and hence $\mathfrak{A}$ is a semi-field plane. sian.

THEOREM 6. If $\mathfrak{A}$ is a TWA-plane of order $p^{2}, p$ a prime with $p \neq 5,11$, then $\mathfrak{A}$ is Desargue-

Proof. If $p=2$ then $\mathfrak{A}$ has order 4 and is therefore the Desarguesian plane of order 4 ; if 
$p=3$ then $\mathfrak{Y}$ is Desarguesian since the only non-Desarguesian translation plane of order 9 is the one over the exceptional nearfield of order 9 and it is not a $W$-plane. Thus we may assume $p>5$. Also, by Theorem 5 we may assume $\mathfrak{A}$ has no homologies with affine axis.

Let $\mathfrak{U}$ be a counterexample to the statement of the theorem, let $G$ be a $W A$-group of $\mathfrak{U}$ and $\mathscr{V}_{\infty}$ the special point of $\mathfrak{A}$ with respect to $G$. Without loss of generality we may assume $G_{\mathscr{0}}$, and hence $G$, contains the group $K$ of $\left(\mathcal{O}, \ell_{\infty}\right)$-homologies since $K$ fixes all the points on $\ell_{\infty}$ and all lines through $\mathcal{O}$. By equation (3.2),

$$
\left|G_{\mathcal{O}}\right|=p^{2}\left(p^{2}-1\right) k
$$

for some integer $k \geqq 1$. Let $p^{s}|| k$. If $E$ is the group of elations of $\mathfrak{A}$ with axis $\mathcal{O} \mathscr{V}_{\infty}$, then $|E| \leqq p^{2}$ and $E$ is contained in a Sylow $p$-subgroup $S$ of $G$ with $|S|=p^{2+s}$. By Theorem $4, G_{\mathcal{O}}$ induces a group $\bar{G}$ of linear transformations on $(Q,+)$ over $G F(p)$ with $\bar{G} \cong G_{\mathscr{O}} / E$; here $(Q,+, \cdot)$ is a quasi-field coordinatizing $\mathfrak{U}$ with $\mathscr{V}_{\infty}=(\infty)$ and $\mathcal{O}=(0,0)$. Also, $\bar{G}$ is transitive on the non-zero vectors of $(Q,+)$ since $G_{0}$ is transitive on the affine points $\neq \mathcal{O}$ of $\mathcal{O} \mathscr{V}_{\infty}$.

$\bar{G}$ is a subgroup of $G L(2, p)$ and $|G L(2, p)|=p\left(p^{2}-1\right)(p-1)$. Thus $|S| E|\leqq p| E \mid,=p^{1+s}$. If $|E|=p^{2}$, then $\mathfrak{A}$ is a semi-field plane and therefore Desarguesian [3, p. 244]. Hence $|E|=p, s=0, p \nmid\left|G_{\mathcal{O}, \mathscr{U}_{\infty}}\right|, \mathscr{U}_{\infty}$ a point on $\ell_{\infty}$ with $\mathscr{U}_{\infty} \neq \mathscr{V}_{\infty}$, and $|\bar{G}|=p\left(p^{2}-1\right) k$.

Assume first that $G_{0}$ is solvable. Then $\bar{G}$ is a solvable transitive group of linear transformations on $(Q,+)$, a vector space of dimension 2 over $G F(p)$. By Huppert [5, pp. 126-7], either (a) $\bar{G} \leqq \Gamma L\left(1, p^{2}\right),|\bar{G}| \mid 2\left(p^{2}-1\right)$ or (b) $p^{2}=5^{2}, 7^{2}, 11^{2}$ or $23^{2}$ and $\bar{G}$ is one of eight possible exceptional groups none of whose orders is divisible by $p$. Thus $p \nmid|\bar{G}|$-a contradiction to the last statement of the previous paragraph. Therefore, $G_{\mathfrak{O}}$ is non-solvable.

By Theorem 3, either (c) $\bar{G}$ contains $S L(2, p)$ and $|\bar{G}|=\frac{1}{2} m p\left(p^{2}-1\right)(p-1)$ with $m \mid 2$ or (d) $\bar{G}$ contains $S L(2,5),|\bar{G}|=60 m(p-1)$ with $m \mid 2$, and $p=11,19,29$ or 59 . (d) cannot happen since it implies $p(p+1) k \mid 60 \mathrm{~m}$. Therefore, (c) holds.

Let $H=G_{0, q_{\infty}}$ and assume first that $H$ is solvable. By Lemma 4, Theorem 4, and the remark following it, $H$ induces a solvable transitive group $\bar{H}$ of linear transformations on $(Q,+)$ as a vector space of dimension 2 over $G F(p)$. Also, $\bar{H} \cong H$ since $H \cap E=I$, and $|H|=p^{-2}\left|G_{\mathscr{O}}\right|=\frac{1}{2} m\left(p^{2}-1\right)(p-1)$ since (c) holds. By [5, pp. 126-7], $|\bar{H}| \mid 4\left(p^{2}-1\right)$. (One of Huppert's exceptional groups has order $4\left(p^{2}-1\right)$, the rest have order $p^{2}-1$ or $2\left(p^{2}-1\right)$.) Thus $\frac{1}{2} m(p-1) \mid 4$; since $m \mid 2$, we must have $p=5$. Since $p=5$ is excluded, $H$ is non-solvable.

Applying Theorem 4 to $\bar{H}$, either (e) $|H|=\frac{1}{2} n p\left(p^{2}-1\right)(p-1)$ with $n \mid 2$, or (f) $|H|=$ $60 n(p-1)$ with $n \mid 2$ and $p=11,19,29$ or 59 . (e) cannot occur; for (e) implies $p|| H \mid=$ $\left|G_{\mathcal{O}, \Psi_{\infty}}\right|$, which is a contradiction. Thus (f) holds, in which case $p=11$ is the only possibility since $|H|=\frac{1}{2} m\left(p^{2}-1\right)(p-1)$. But $p=11$ is excluded. Hence the counterexample $\mathfrak{I}$ does not exist.

REmark. Note that in the two excluded cases, $p=5$ or 11 , the above proof gives the following information about a possible counterexample to the theorem. The group $G_{\mathscr{O}}$ (and hence $G$ ) is non-solvable, the group $E$ of elations with axis $\mathcal{O} \mathscr{V}_{\infty}$ has order $p$, and $G_{\mathscr{O}} / E \cong$ $S L(2, p)$. For $p=5$ the group $G_{\mathcal{O}, \mathscr{U}_{\infty}}$ is solvable, but for $p=11, G_{\mathscr{O}, \mathscr{U}_{\infty}} \cong S L(2,5)$.

Also, for $p=5$ there is at least one counterexample, the plane $W(5)$, but for $p=11$ no counterexample is known. 


\section{WA-planes with solvable $W A$-groups.}

LEMMA 5. Let $\mathfrak{A}$ be a TWA-plane of order $p^{r}$ with $W A$-group $G$ and special point $\mathscr{V}_{\infty}$. If $\mathcal{O}$ is an affine point of $\mathfrak{U}$ and $\mathscr{U}_{\infty}$ a point of $\ell_{\infty}$ with $\mathscr{U}_{\infty} \neq \mathscr{V}_{\infty}$, then $G_{0}=S G_{0, \mathscr{q}_{\infty}}, S$ a $S y$ low p-subgroup of $G_{0}$, and $S$ is transitive on $l_{\infty}-\left\{\mathscr{V}_{\infty}\right\}$.

Proof. By equation (3.2), $\left|G_{\mathcal{O}}\right|=p^{r}\left|G_{\mathcal{O}, q_{\infty}}\right|$. If $p^{s}||\left|G_{\mathcal{O}, q_{\infty}}\right|$, then $|S|=p^{r+s}$. Consider $S_{\mathscr{U}_{\infty}}$. Now, $|S|=\left|S_{\mathscr{U}_{\infty}}\right||\Omega|$, where $\Omega$ is the orbit of $S$ containing $\mathscr{U}_{\infty}[12, \mathrm{p} .5]$. Since $S_{\mathscr{Q}_{\infty}} \leqq G_{0, \mathscr{U}_{\infty}},\left|S_{\mathscr{q}_{\infty}}\right| \mid p^{s}$ and thus $p^{r}|| \Omega \mid$. This implies $\Omega=l_{\infty}-\left\{\mathscr{V}_{\infty}\right\}$. Therefore, $S$ is transitive on $l_{\infty}-\left\{\mathscr{V}_{\infty}\right\}$ and it follows that $G_{\mathscr{O}}=S G_{\mathcal{O}, \mathscr{q}_{\infty}}$.

THEOREM 7. Let $\mathfrak{A}$ be a TWA-plane of order $p^{r} \neq 5^{2}, 2^{6}, 3^{4}, G$ a WA-group of $\mathfrak{U}$, and $\mathscr{V}_{\infty}$ the special point of $\mathfrak{I}$ with respect to $G$. If there is a point $\mathscr{U}_{\infty} \in l_{\infty}$ with $\mathscr{U}_{\infty} \neq \mathscr{V}_{\infty}$ such that $G_{\mathscr{U}_{\infty}}$ is solvable and $G$ contains a non-trivial elation with affine axis $\downarrow$ through $\mathscr{V}_{\infty}$, then $\mathfrak{A}$ is a semi-field plane.

Proof. Let $\mathcal{O}$ be an affine point on $l$, let $(Q,+, \cdot)$ be a quasi-field coordinatizing $\mathfrak{I}$ with $\mathscr{V}_{\infty}=(\infty), \mathcal{O}=(0,0), \mathscr{U}_{\infty}=(0)$, and let $E$ be the group of elations with axis $l=\mathcal{O} \mathscr{V}_{\infty}$. Note that $E$ is a subgroup of $G_{0}$. Because of Theorems 5 and 6 we may assume $G_{\mathscr{O}}$ has no homologies with axis $O \mathscr{V}_{\infty}$ and that $r>2$. (The case $p^{r}=11^{2}$ is handled by the remark after Theorem 6.)

Consider the group $G_{\mathcal{O}, \mathscr{U}_{\infty}}$; this group is solvable and transitive on the affine points of $\mathcal{O} \mathscr{V}_{\infty}$ other than $\mathcal{O}$ (Lemma 4). It follows that $G_{\mathcal{O}, \mathscr{q}_{\infty}}$ induces a solvable transitive group $\bar{H}$ of linear transformations on $(Q,+)$ over $G F(p)$. Also, $\bar{H} \cong G_{\mathcal{O}, q_{\infty}}$ by the last statement of the previous paragraph. Since $r>2$ and $p^{r} \neq 3^{4}, \bar{H}$ is a subgroup of $\Gamma L\left(1, p^{r}\right)$ [5, pp. 126-7]. Therefore, $G_{\mathcal{O}, q_{\infty}}$ is a subgroup of $\Gamma L\left(1, p^{r}\right)$.

By equation (3.3), $\left|G_{\mathcal{O}, \mathscr{q}_{\infty}}\right|=\left(p^{r}-1\right)\left|G_{\mathfrak{O}, \mathscr{P}, \mathfrak{u}_{\infty}}\right|$. Since $p^{r} \neq 2^{6}$ and $r>2, p^{r}-1$ has a prime $p$-primitive divisor $u$ (Zsigmondy [13]); i.e. $u \mid\left(p^{r}-1\right)$ but $u \times\left(p^{i}-1\right)$ for $1 \leqq i<r$. If $u^{a} \|\left(p^{r}-1\right)$, then $G_{0, q_{\infty}}$ has a Sylow $u$-subgroup $T$ of order $u^{a}$ and $T$ is a cyclic characteristic subgroup of $G_{\mathcal{O}, q_{\infty}}\left[8\right.$, Lemma 2.4]. Thus $G_{\mathcal{O}, q_{\infty}} \leqq N$, the normalizer of $T$ in $G_{0}$.

Let $\tau \in T$. Since $E \triangleleft G_{\mathcal{O}}$, the mapping $M_{\tau}$ defined by $\rho M_{\tau} \equiv \tau^{-1} \rho \tau, \rho \in E$, is an automorphism of $E$ having order $u^{j}$ for some integer $j \geqq 0$ (since $\tau$ has order $u^{k}$ ). If $|E|=p^{s}$, then the automorphism group of $E$ has order $m=p^{n} \prod_{i=1}^{s}\left(p^{i}-1\right), n=\frac{1}{2} s(s-1)$, for $E$ is an elementary abelian $p$-group. If $T$ has an element $\tau$ such that $M_{\tau}$ has order $u^{j}, j>0$, then $u \mid m$; this implies $s=r$. It follows that $|E|=p^{r}, \mathfrak{A}$ is $\left(\mathscr{V}_{\infty}, \mathcal{O} \mathscr{V}_{\infty}\right)$-transitive, and hence $\mathfrak{U}$ is a semi-field plane.

If $M_{\tau}$ has order 1 for all $\tau \in T$, then $\rho \tau=\tau \rho$ for all $\rho \in E$ and for all $\tau \in T$. Thus $E \leqq N$ and $N$ has order at least $p^{s}\left(p^{r}-1\right)\left|G_{0}, \mathscr{P}, \mathscr{q}_{\infty}\right|$. Thus the index $\left[G_{0}: N\right]$ divides $p^{r-s}$; this implies $\left[G_{\mathcal{O}}: N\right]=1$ (for it has to be congruent to 1 modulo $u$ ) and hence $T \triangleleft G_{\mathcal{O}}$. Now, $T$ fixes the point $\mathscr{U}_{\infty}$ on $l_{\infty}$. Since $T \triangleleft G_{0}, G_{0}$ permutes the fixed points of $T$ on $l_{\infty}$ among themselves. Thus, $G_{\mathcal{O}}$ transitive on $\ell_{\infty}-\left\{\mathscr{V}_{\infty}\right\}$ implies $T$ fixes all the points of $l_{\infty}$ and hence $T$ consists of homologies with centre $\mathcal{O}$ and axis $l_{\infty}$. Since the group of $\left(\mathcal{O}, l_{\infty}\right)$-homologies of $\mathfrak{A}$ has order $p^{j}-1$ for some $j$ with $1 \leqq j \leqq r\left[3\right.$, p. 132], and $|T|=u^{a}$, we have $u^{a} \mid\left(p^{j}-1\right)$. Because $u$ is a $p$-primitive divisor, we have $j=r$. This implies $\mathfrak{A}$ is Desarguesian $[3$, p. 132]. 
We come now to the main result of this section.

THEOREM 8. Let $\mathfrak{A}$ be a TWA-plane of order $p^{r} \neq 2^{6}$, p a prime, with a WA-group $G$. If $G$ is solvable then $\mathfrak{A}$ is a semi-field plane.

Proof. Let $\mathscr{V}_{\infty}$ be the special point of $\mathfrak{U}$ with respect to $G$ and coordinatize $\mathfrak{A}$ by a quasifield $(Q,+, \cdot)$ with $\mathscr{V}_{\infty}=(\infty), \mathcal{O}=(0,0), \mathscr{U}_{\infty}=(0)$. By Theorem 5 we may assume that $\mathfrak{A}$ has no homologies with affine axis. In particular, we may assume there are no homologies with axis $\mathcal{O} \mathscr{V}_{\infty}$. By Theorem 6 and the remark following it, we may assume $r>2$.

Assume first that $p^{r}=3^{4}$. Since $G_{0}$ is solvable, the group $\bar{G}$ of linear transformations induced on $(Q,+)$ by $G_{\mathcal{O}}$ is solvable. By $[5, \mathrm{pp} .126-7]$, either $\bar{G} \leqq \Gamma L\left(1,3^{4}\right)$ or $\bar{G}$ is one of three groups given in [5]. In all four cases $3 \nmid|\bar{G}|$. Since $\bar{G}=G_{\mathcal{o}} / E, E$ the group of elations with axis $\mathcal{O} \mathscr{V}_{\infty}$, we have $|E|=3^{4}$ and $\mathfrak{U}$ is a semi-field plane.

Assume now that $p^{r} \neq 3^{4}$. We claim that $G_{0}$ contains non-trivial elations with axis $O \mathscr{V}_{\infty}$. For if not, the group $\bar{G}$ of linear transformations induced on $(Q,+)$ by $G_{\mathscr{O}}$ is isomorphic to $G_{\mathcal{O}}$. Since $\bar{G}$ is solvable, $\bar{G} \leqq \Gamma L\left(1, p^{r}\right)$ by $\left[5\right.$, pp. 126-7], and thus $|\bar{G}|=\left|G_{\mathcal{O}}\right|$ divides $r\left(p^{r}-1\right)$. Since $p^{r}|| G_{o} \mid$ by equation (3.2), we have $p^{r} \mid r$, which is a contradiction. Thus $G_{\mathscr{o}}$ has nontrivial elations with axis $\mathcal{O \mathscr { V } _ { \infty }}$. The theorem now follows from the previous theorem.

CoRollary 8.1. If $\mathfrak{A}$ is a TWA-plane of order $p^{r}, p$ an odd prime, and $\mathfrak{A}$ has odd dimension over its kernel, then $\mathfrak{A}$ is a semi-field plane.

Proof. Let $G$ be a $W A$-group of $\mathfrak{A}$ and $\mathscr{V}_{\infty}$ the associated special point on $l_{\infty}$. By Hering [4, paragraph preceding Corollary 1], the stabilizer of $\mathscr{V}_{\infty}$ in the full collineation group of $\mathfrak{U}$ is solvable. Thus $G$ is solvable and the corollary follows from Theorem 8 .

COROLLARY 8.2. Let $\mathfrak{A}$ be a WA-plane of odd order $p^{r}$ with WA-group $G$. $\mathfrak{A}$ is a semi-field plane if one of the following conditions holds:

(1) $G$ is solvable,

(2) $p^{r}$ is a non-square.

Proof. By Theorem 1, $\mathfrak{A}$ or its dual $\mathfrak{A}^{\prime}$ is a $T W A$-plane. It then follows from either Theorem 8 or Corollary 8.1 that $\mathfrak{A}$ or $\mathfrak{A}^{\prime}$ is a semi-field plane. Since the dual of a semi-field plane is a semi-field plane, the corollary follows.

5. WA-planes of dimension 2 over their kernel. Let $\mathfrak{A}$ be a $T W A$-plane of order $q^{2}$, $q=p^{r} \neq 5,11,2^{3}, 3^{2}$ with $p$ a prime and $r \geqq 1$, and having kernel $\bar{K}=G F(q)$. Let $G$ be a $W A$-group of $\mathfrak{U}$ and $\mathscr{V}_{\infty}$ the associated special point. Note that without loss of generality we may assume $G$ contains the group $K$ of $\left(\mathcal{O}, l_{\infty}\right)$-homologies, $I_{\infty} \mathcal{O}$ an affine point. As in section 3, we get the equations:

$$
\begin{aligned}
\left|G_{\mathcal{O}}\right| & =q^{2}\left|G_{\mathcal{O}, q_{\infty}}\right|=q^{2}\left(q^{2}-1\right) t, \\
\left|G_{O, q_{\infty}}\right| & =\left(q^{2}-1\right) t,
\end{aligned}
$$

where $\mathscr{U}_{\infty} \in l_{\infty}, \mathscr{U}_{\infty} \neq \mathscr{V}_{\infty}$, and $t$ is a positive integer. By Theorem 5 we may assume there are no homologies with affine axis and by Theorem 6 we may assume $q$ is not a prime. By 
Theorems 7 and 8 we may assume that both $G_{\mathcal{O}}$ and $G_{\mathcal{O}} \mathscr{U}_{\infty}$ are non-solvable, for $G=T G_{\mathcal{O}}$ and $G_{\mathscr{U}_{\infty}}=T G_{\mathcal{O}, \mathscr{U}_{\infty}}$ where $T$ is the group of translations.

If $\bar{G}$ and $\bar{H}$ are the permutation groups induced on $\mathcal{O} \mathscr{V}_{\infty}$ by $G_{\mathcal{O}}$ and $G_{\mathcal{O}}, \mathscr{U}_{\infty}$ respectively, then $\bar{G}=G_{\mathscr{O}} / E$ and $\bar{H}=G_{\mathcal{O}, \mathscr{U}_{\infty}} / E$ with $E$ the group of elations with axis $\mathcal{O} \mathscr{V}_{\infty}$. By Theorem 3 , we have $\bar{G} \leqq \Gamma L(2, q)$; since $q^{2} \chi|\Gamma L(2, q)|$, it follows from equation (5.1) that $p|| E \mid$. Also, by Theorem 3 , we have

$$
|\bar{H}|=\frac{1}{2} k q\left(q^{2}-1\right)(q-1)
$$

with $k \mid 2 r$. It follows that $q|| G_{\mathcal{O}, \mathscr{U}_{\infty}} \mid$ and hence $q^{3}|| G_{\mathcal{O}} \mid$. Applying Theorem 3 to $\bar{G}$ we have

$$
|\bar{G}|=\frac{1}{2} m q\left(q^{2}-1\right)(q-1)
$$

with $m \mid 2 r$. It follows that $q p^{r-s}|| E \mid$ where $p^{s}|| r$. Note that $s<r$. Hence $|E|>q$.

Let $H=G_{O, q_{\infty}}$; then $|H|=\left(q^{2}-1\right) t$ by equation (5.2). Because $q$ is not a prime and $q \neq 2^{3}$, the integer $q^{2}-1$ has a prime $p$-primitive divisor $u$ [13]. Choose an element $\tau \in H$ with $|\tau|=u$. Because $E \triangleleft G_{\mathcal{O}}, \tau$ induces an automorphism $M_{\tau}: E \rightarrow E$ given by $\rho M_{\tau}=\tau^{-1} \rho \tau$. For every $\tau \in H$ with $|\tau|=u$, we have two possibilities: (1) $\left|M_{\tau}\right|=u$, or (2) $\left|M_{\tau}\right|=1$. If $\left|M_{\tau}\right|=u$ for some $\tau$, then it follows as in the proof of Theorem 7 that $\mathfrak{U}$ is a semi-field plane.

Assume $\left|M_{\tau}\right|=1$ for all elements $\tau \in H$ with $|\tau|=u$. Let $\tau$ be one such element in $H$. It follows that the elements of $E$ commute with $\tau$ and hence $E$ permutes the fixed points of $\tau$ among themselves. Since $\tau$ fixes $\mathscr{U}_{\infty}, \mathfrak{B} \equiv\left\{\mathscr{U}_{\infty} \rho \mid \rho \in E\right\} \cup\left\{\mathscr{V}_{\infty}\right\}$ is a set of fixed points of $\tau$. Furthermore, the components of $\mathfrak{A}$ of the form $\mathscr{O P} \mathscr{P}_{\infty}, \mathscr{P}_{\infty} \in \mathfrak{B}$, lie in the same Desarguesian net $\mathfrak{N}$ (Ostrom [9, Theorem 3.3 and its proof]); by Ostrom [9, (4.7) on p. 25] every collineation $\tau$ of $H$ having order $u$ is a collineation of Desarguesian plane $\mathfrak{A}$ containing the net $\mathfrak{P}$. Since $\tau$ fixes more than $q+1$ points of $\vec{l}_{\infty}$, the line at infinity of $\overrightarrow{\mathfrak{I}}, \tau$ is in the group $L$ of $\left(\mathcal{O}, \bar{l}_{\infty}\right)$-homologies of $\mathfrak{A}$. Thus $\langle\tau\rangle$ is the unique cyclic subgroup of order $u$ in $L$ since $L$ is cyclic.

If $\tau$ is an element of order $u$ in $H$ and $\alpha \in H$, then $\alpha^{-1} \tau \alpha$ is an element of order $u$ in $H$; by the previous paragraph, $\alpha^{-1} \tau \alpha$ and $\tau$ are elements of order $u$ in $L$ and thus $\alpha^{-1} \tau \alpha \in\langle\tau\rangle$. Hence $\langle\tau\rangle \triangleleft H$. If $N$ is the normalizer of $\langle\tau\rangle$ in $G_{\Theta}$, then $E \leqq N$ and $H \leqq N$. Since $q p^{r-s}\left(q^{2}-1\right) t=$ $|E H|$ and $q^{2}\left(q^{2}-1\right) t=\left|G_{0}\right|$, we have $\left[G_{\mathscr{O}}: N\right] \mid p^{s}$ and $s<r$. It follows as in the last paragraph of the proof of Theorem 7 that $N=G_{\mathscr{O}}$, that $\langle\tau\rangle \triangleleft G_{\mathscr{\ell}}$, and that $\mathfrak{A}$ is Desarguesian.

Thus we have proved the following theorem.

THEOREM 9. If $\mathfrak{U}$ is a TWA-plane of order $q^{2}, q \neq 5,11,2^{3}, 3^{2}$, and having kernel containing $G F(q)$, then $\mathfrak{A}$ is a semi-field plane.

CoROLlaRY 9.1. If $\mathfrak{Q}$ is a WA-plane of order $q^{2}, q$ odd and $q \neq 5,11,3^{2}$, having kernel containing $G F(q)$, then $\mathfrak{U}$ is a semi-field plane.

Acknowledgement. The author wishes to thank the referee for his many suggestions concerning style and wording.

\section{REFERENCES}

1. A. A. Albert, On the collineation groups associated with twisted fields, Calcutta Math. Soc. Golden Jubilee Commemoration volume, Part II (1958/59), 485-497.

2. J. Andre', Über Perspektivitäten in endlichen projektiven Ebenen, Arch. Math. (Basel) 6 (1954), 29-32. 
3. P. Dembowski, Finite Geometries (Springer-Verlag, 1968).

4. C. Hering, On 2-groups operating on projective planes, Illinois J. Math. 16 (1972), 581-595. 126-150.

5. B. Huppert, Zweifach transitive, auflösbare Permutations-gruppen, Math. Z. 68 (1957),

6. N. J. Johnson and M. J. Kallaher, Transitive collineation groups on affine planes, Math. $Z$. 135 (1973), 149-164.

7. M. J. Kallaher, Rank 3 affine planes of square order, Geometriae Dedicata 1 (1973), 415-425.

8. M. J. Kallaher and T. G. Ostrom, Fixed-point-free linear groups, rank three planes, and Bol quasi-fields, J. Algebra 18 (1971), 159-178.

9. T. G. Ostrom, Finite Translation Planes (Springer-Verlag, 1971).

10. T. G. Ostrom, Classification of finite translation planes, Proc. Internat. Confer. on Projective Planes, Pullman, WA. (1973), 195-213.

11. M. Walker, On translation planes and their collineation groups, Thesis, Westfield College, University of London (1973).

12. H. Wielandt, Finite Permutation Groups (Academic Press, 1964).

13. K. Zsigmondy, Zur Theorie der Potenzreste, Monatsh. Math. Phys. (1892), 265-284.

\section{UNIVERSITÄT KAISERSLAUTERN \\ WEST GERMANY}

